\title{
Role of H-bond formation in the photoreactivity of curcumin*
}

\author{
Luca Nardo $^{\mathrm{a}, * *}$, Roberta Paderno ${ }^{\mathrm{a}}$, Alessandra Andreoni ${ }^{\mathrm{a}}$, Már Másson ${ }^{\mathrm{b}}$, Tone Haukvik ${ }^{\mathrm{c}}$ \\ and Hanne Hjorth Tønnesen ${ }^{\mathrm{c}}$ \\ ${ }^{a}$ CNISM-INFM-CNR and Department of Physics and Mathematics, University of Insubria at Como, \\ Como, Italy \\ ${ }^{\mathrm{b}}$ Faculty of Pharmacy, University of Iceland, Hagi, Reykjavik, Iceland \\ ${ }^{\mathrm{c}}$ School of Pharmacy, University of Oslo, Oslo, Norway
}

\begin{abstract}
Curcumin is the main constituent of curry. In its ground state it shows chemo-preventive, chemo-therapeutic and anti-inflammatory effects. For its immunostimulating action it has been considered for the development of drugs suitable for treating AIDS and cystic fibrosis. Further biological action is induced in curcumin by photoactivation: in suitable environmental conditions electronically excited curcumin can act as a singlet oxygen generator. Moreover, cytotoxicity is enhanced by light exposure and antibacterial effects are photosensitized. This work is aimed to understand the photobiological action of curcumin by elucidating the deactivation mechanisms of its first excited singlet state. In particular we find evidence of the role of tautomerization in the excited state by measuring fluorescence lifetimes and quantum yields for such compound dissolved in solvents of different polarity and H-bonding capability. Degradation quantum yield and singlet oxygen generation efficiency were also measured in acetonitrile and methanol. The results emphasize the strong dependence of the deactivation processes from the environment. The deactivation phenomenology can be fully explained by postulating intramolecular proton transfer in the $c i s$ enol conformer to be the leading non-radiative deactivation pathway.
\end{abstract}

Keywords: Curcumin, photosensitizer, excited state intramolecular proton transfer

\section{Introduction}

Curcumin, the yellow-orange pigment of curry spice, is isolated from the rhizome of Curcuma longa $L$. Commercially available curcumin is a mixture of three curcuminoids, namely, curcumin, demethoxy- and bisdemethoxy-curcumin, the latter two amounting to nearly $30 \%$ in the samples labeled "pure" [1].

Curcumin in the ground state exhibits chemo-preventive, chemo-therapeutic and anti-inflammatory activities. It is also considered as either a drug or model substance for the treatment of AIDS and cystic fibrosis, as well as an immunostimulating agent [2-5]. Some of these biological effects are enhanced upon excitation with light [6], in which case curcumin can generate singlet oxygen in a strongly solventdependent fashion and act as a scavenger of hydroxyl radicals [7-9].

\footnotetext{
* Studies on curcumin and curcuminoids XXXII.

** Corresponding author: Luca Nardo, CNISM-INFM-CNR and Department of Physics and Mathematics, University of Insubria at Como, Via Valleggio, 11-22100 Como, Italy. Tel.: +39 031 2386272; Fax: +39 031 2386119; E-mail: luca.nardo@uninsubria.it.
} 
A

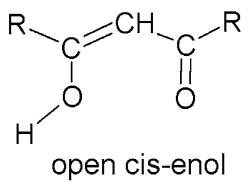<smiles>[R]C1=COPC([R])=C1</smiles><smiles>[R]C1=CC([R])OCO1</smiles>

closed cis-enol<smiles>[R]C(=O)C=C([R])O</smiles><smiles>[R]C(=O)C=C([R])O</smiles><smiles>[R]C(=O)C=C([R])O</smiles>
trans-enol
B<smiles>[R]C(=O)CC([R])=O</smiles><smiles>[R]C(=O)CC([R])=O</smiles><smiles>[R]C(=O)CC([R])=O</smiles>

trans diketo
C<smiles>[R]C1=COPC([R])=C1</smiles>

$\mathrm{H}$ bonded cis-enol<smiles>[R]C(=CC)OB1OC(C)=C([R])C=C1[R]</smiles>

In the case of curcumin $\mathrm{R}$ is<smiles>C=Cc1ccc(O)c(C)c1</smiles>

Fig. 1. (A) Curcumin enol isomers; (B) curcumin diketo isomers; (C) intramolecular hydrogen bonding and excited singlet state deactivation through excited-state intramolecular proton-transfer (ESIPT) in the cis enol conformer.

Curcumin belongs to the group of $\beta$-diketones and exhibits tautomerism between enol- and ketostructures, as shown in Fig. 1 (A and B, respectively). The enol form is characterized by a strong intramolecular hydrogen bond (H-bond) (see Fig. 1C). Moreover, in solution curcumin can form intermolecular H-bonds with the solvent molecules and this strongly influences its physicochemical properties in both the ground and excited states. Hydrogen bonding and charge delocalization influence the drug excited-state interactions with biomolecules, thus its therapeutic potential.

In the present work we investigate the excited-state behavior of curcumin in various media, with emphasis on inter- and intramolecular H-bonding, to optimize the structure of systems to be used in clinical applications for the delivery of a curcumin-based photosensitized drug. To this purpose the absorption and fluorescence spectra of highly pure synthetic curcumin were measured in a number of organic solvents differing as to polarity and $\mathrm{H}$-bonding capability. In each solvent the fluorescence quantum yield was determined and time resolved fluorescence measurements in the picosecond time domain were performed. Finally we determined the singlet oxygen formation capability and the photodegradation quan- 
tum yield in two of the solvents. The data were interpreted by postulating excited-state intramolecular proton transfer to be the leading non-radiative deactivation pathway, by analogy to the well known phenomenology of other $\beta$-diketones.

\section{Materials and methods}

\subsection{Chemicals and sample preparation}

Curcumin was synthesized as previously described [10]. All the solvents were of purity $\geqslant 99.5 \%$ and were used as received, except ethyl acetate, which was dried over sodium sulfate. Samples in organic solvents were prepared the same day they were used for measurements.

\subsection{UV-VIS absorption spectra}

The UV-VIS absorption spectra were measured by a Shimadzu UV-2401 PC UV-VIS recording spectrophotometer.

\subsection{Fluorescence spectra and quantum yields}

Steady-state fluorescence measurements were carried out on a PTI modular fluorescence system. The excitation source was a $75 \mathrm{~W}$ xenon lamp and the monochromators are Model 101 with $\mathrm{f} / 4$ 0.2-m Czerny-Turner configuration, whose entrance and exit slits were adjusted to $2 \mathrm{~nm}$. Spectral correction of the emission was automatically performed. The samples, having peak absorbance values $<0.1$ and being thermostated at $25.0 \pm 0.1^{\circ} \mathrm{C}$, were excited at their respective absorption maxima.

The fluorescence quantum yields were determined from the spectrum integrated fluorescence by using, as a reference value, that of quinine sulfate in $0.05 \mathrm{M} \mathrm{H}_{2} \mathrm{SO}_{4}$ excited at its $344 \mathrm{~nm}$ absorption peak: $\Phi_{\text {Ref }}=0.51$ [11]. The calculated quantum yields were corrected for differences in peak absorbance and in refractive index of the solutions.

\subsection{Fluorescence decay measurements}

The fluorescence of the solutions, which were contained in a $1 \times 1 \mathrm{~cm}^{2}$ fluorimeter quartz cuvette, was excited at $420 \mathrm{~nm}$ by the second harmonic (SH) output of a SESAM mode locked Ti:sapphire laser (Tiger-ps SHG, Time Bandwidth Products, Zurich, $\mathrm{CH}$ ) operating at $48 \mathrm{MHz}$ repetition rate. The typical SH output power was $>20 \mathrm{~mW}$. The pulse width (measured at $840 \mathrm{~nm}$ ) was 3.9 ps. The fluorescence at $\lambda>500 \mathrm{~nm}$ was collected at $90^{\circ}$ to the excitation beam through a cut-off filter (LL-500, Corion, Holliston, MA) by a $40 \times$ microscope objective and focused onto the sensitive area of the detector. This was a single-photon avalanche diode with built-in active quenching circuitry (PDM50 micro-photondevices, Bolzano, IT) that is used in a single-photon timing apparatus. The time-to-amplitude converter (TAC) of such apparatus is started by the PDM50 output and stopped by the re-shaped output of a fast photodiode monitoring the excitation pulses. Pulse-height spectra of the TAC output are measured with a multichannel analyzer and cover a time range of about $50 \mathrm{~ns}$ with the resolution of $5.35 \mathrm{ps} / \mathrm{channel}$. All fluorescence decays were collected up to 10,000 peak counts in strict single photon regime by suitably attenuating the excitation beam. At the laser excitation wavelength $(420 \mathrm{~nm})$, the solutions absorbance was $<0.05$. 
The fluorescence decay data were fitted, without deconvolving the system pulse response (full-width at half-maximum duration $\sim 30 \mathrm{ps}$ ), either with single, double, or triple exponentials above a constant background, by minimizing the chi-square value through a Levenberg-Marquardt algorithm. Three decay curves were acquired for each solution. The mean of the values obtained from the corresponding fits, with errors given by the standard deviations, were assumed as the time constant, $\tau_{i}$, and initial relative amplitude, $A_{i}$, of the $i$ th decay component, being the $A_{i}$ values calculated at the peak channel of the experimental data.

\subsection{Luminescence detection of singlet oxygen}

The system described in section 2.3 was equipped with an EQ-817 Germanium Detection system operated under liquid nitrogen conditions. The generation of singlet oxygen $\left({ }^{1} \mathrm{O}_{2}\right)$ was measured by steady-state detection of luminescence at $1270 \mathrm{~nm}$. An average of 5 luminescence spectra was used to calculate the area under the emission band from $1065 \mathrm{~nm}$ to $1550 \mathrm{~nm}$.

\subsection{Degradation quantum yields}

The reaction quantum yield of selected samples was measured using the potassium ferrioxalate chemical actinometer [12]. The samples were irradiated by using a monochromator (Applied Photophysics Ltd., f 3.4, $900 \mathrm{~W}$ xenon arc lamp) operated with a bandwidth of $20 \mathrm{~nm}$ at the selected wavelength. The number of sample molecules reacted per unit time and per unit volume as a function of exposure time was quantified by means of reversed phase HPLC. The separation was performed on a $150 \times 3.9 \mathrm{~mm}$ Nova Pak ${ }^{\circledR} \mathrm{C}_{18}$ column (Waters, Milford, USA). The mobile phase was a mixture of acetonitrile and $0.5 \%$ citric acid adjusted to $\mathrm{pH} 3$ with $\mathrm{KOH}$. The residual curcumin after exposure was quantified by measuring its absorption at $350 \mathrm{~nm}$, in order to observe tentative degradation products. The chromatic system consisted of a Shimazu LC-9A pump, a Shimadzu SP D-10A UV-VIS detector, a Shimadzu SIL-10 DV auto sampler and a Shimadzu C-R3A integrator.

\section{Results}

The solvents used in the present study can be divided into four main categories; non-polar (cyclohexane), polar non-protic (chloroform, ethyl acetate, acetone, acetonitrile), polar H-bond accepting (dimethylformamide, DMFA, and dimethylsulfoxide, DMSO), and polar H-bond donating (isopropanol, ethanol, methanol, ethylene glycol). The data are summarized in Table 1.

\subsection{Absorption spectra}

The absorption spectra mainly consist of broad and essentially structureless bands (see Fig. 2), even if the spectra in protic solvents display a weak shoulder, which is also evident in ethyl acetate. Only in cyclohexane the spectrum definitely exhibits two bands. The nature of the solvent slightly affects the wavelength of maximum absorption, $\lambda_{\mathrm{Abs}}$. A small red shift is observed on going from cyclohexane to more polar solvents (see the $\lambda_{\text {Abs }}$ values in Table 1 and Fig. 2). The largest red shifts are observed for hydrogen bonding solvents. A similar trend in the absorption peak wavelength has been reported by other authors [9]. 
Table 1

Selected spectroscopic properties of curcumin

\begin{tabular}{|c|c|c|c|c|c|c|c|c|c|c|}
\hline Solvent & $\begin{array}{l}\lambda_{\mathrm{Abs}} \\
(\mathrm{nm})\end{array}$ & $\begin{array}{c}\lambda_{\mathrm{Fl}} \\
(\mathrm{nm})\end{array}$ & $\Phi_{\mathrm{Fl}}$ & $\begin{array}{c}\tau_{1}(\mathrm{ps})^{*} \\
\left(A_{1}\right)\end{array}$ & $\begin{array}{c}\tau_{2}(\mathrm{ps})^{*} \\
\left(A_{2}\right)\end{array}$ & $\begin{array}{c}\tau_{3}(\mathrm{ps})^{*} \\
\left(A_{3}\right)\end{array}$ & $\begin{array}{c}\tau_{\mathrm{av}} \\
(\mathrm{ps})^{* *}\end{array}$ & $\begin{array}{c}k_{\mathrm{Fl}} \\
\left(10^{9} \mathrm{~s}^{-1}\right)\end{array}$ & $\begin{array}{c}k_{\mathrm{NR}} \\
\left(10^{9} \mathrm{~s}^{-1}\right)\end{array}$ & $\Phi_{\text {Degr }}$ \\
\hline \multicolumn{11}{|l|}{ Nonpolar } \\
\hline Cyclohexane & 408,429 & $502,471,443$ & $0.006 \pm 0.001$ & $57 \pm 1(0.83)$ & $256 \pm 3(0.16)$ & $\begin{array}{c}1405 \pm 78 \\
(<0.01)\end{array}$ & 89 & 0.067 & 11.17 & \\
\hline \multicolumn{11}{|l|}{ Polar, nonprotic } \\
\hline Chloroform & 419 & 503 & $0.094 \pm 0.005$ & $596 \pm 4(1)$ & & & 596 & 0.16 & 1.51 & \\
\hline Ethyl acetate & 419 & 494 & $0.105 \pm 0.001$ & $467 \pm 3(1)$ & & & 467 & 0.22 & 1.92 & \\
\hline Acetone & 420 & 510 & $0.174 \pm 0.006$ & $702 \pm 4(1)$ & & & 702 & 0.25 & 1.42 & \\
\hline Acetonitrile & 419 & 521 & $0.156 \pm 0.003$ & $695 \pm 2(1)$ & & & 695 & 0.22 & 1.22 & $0.061 \pm 0.011$ \\
\hline \multicolumn{11}{|c|}{ Hydrogen bond acceptors } \\
\hline DMFA & 431 & 536 & $0.041 \pm 0.001$ & $246 \pm 3(>0.98)$ & $708 \pm 98(<0.02)$ & & 251 & 0.16 & 3.82 & \\
\hline DMSO & 434 & 550 & $0.026 \pm 0.002$ & $117 \pm 4(0.79)$ & $299 \pm 13(0.21)$ & $\begin{array}{c}2150 \pm 110 \\
(<0.002)\end{array}$ & 155 & 0.17 & 6.28 & \\
\hline \multicolumn{11}{|c|}{ Hydrogen bond donors } \\
\hline Isopropanol & 431 & 545 & $0.114 \pm 0.004$ & $476 \pm 1(1)$ & & & 476 & 0.24 & 2.10 & \\
\hline Ethanol & 430 & 553 & $0.033 \pm 0.004$ & $221 \pm 6(0.89)$ & $579 \pm 10(0.11)$ & & 260 & 0.13 & 3.72 & \\
\hline Methanol & 423 & 566 & $0.028 \pm 0.002$ & $138 \pm 5(0.85)$ & $302 \pm 11(0.15)$ & & 162 & 0.17 & 6.00 & $0.021 \pm 0.010$ \\
\hline Ethylene glycol & 433 & 566 & $0.022 \pm 0.004$ & $188 \pm 7(0.73)$ & $350 \pm 10(0.27)$ & & 232 & 0.95 & 3.36 & \\
\hline
\end{tabular}

Absorption peak wavelength, $\lambda_{\mathrm{Abs}}$; wavelength of maximum fluorescence, $\lambda_{\mathrm{Fl}}$; fluorescence quantum yield, $\Phi_{\mathrm{Fl}}$; fluorescence decay times, $\tau_{i}$, and relative amplitudes, $A_{i}$; average fluorescence lifetime, $\tau_{\mathrm{av}}$, calculated by disregarding the decay components accounting for less than $5 \%$ of the fluorophores; fluorescence decay rate, $k_{\mathrm{Fl}} ;$ non-radiative decay rate, $k_{\mathrm{NR}}$, and photodegradation quantum yield, $\Phi_{\mathrm{Degr}}$. 


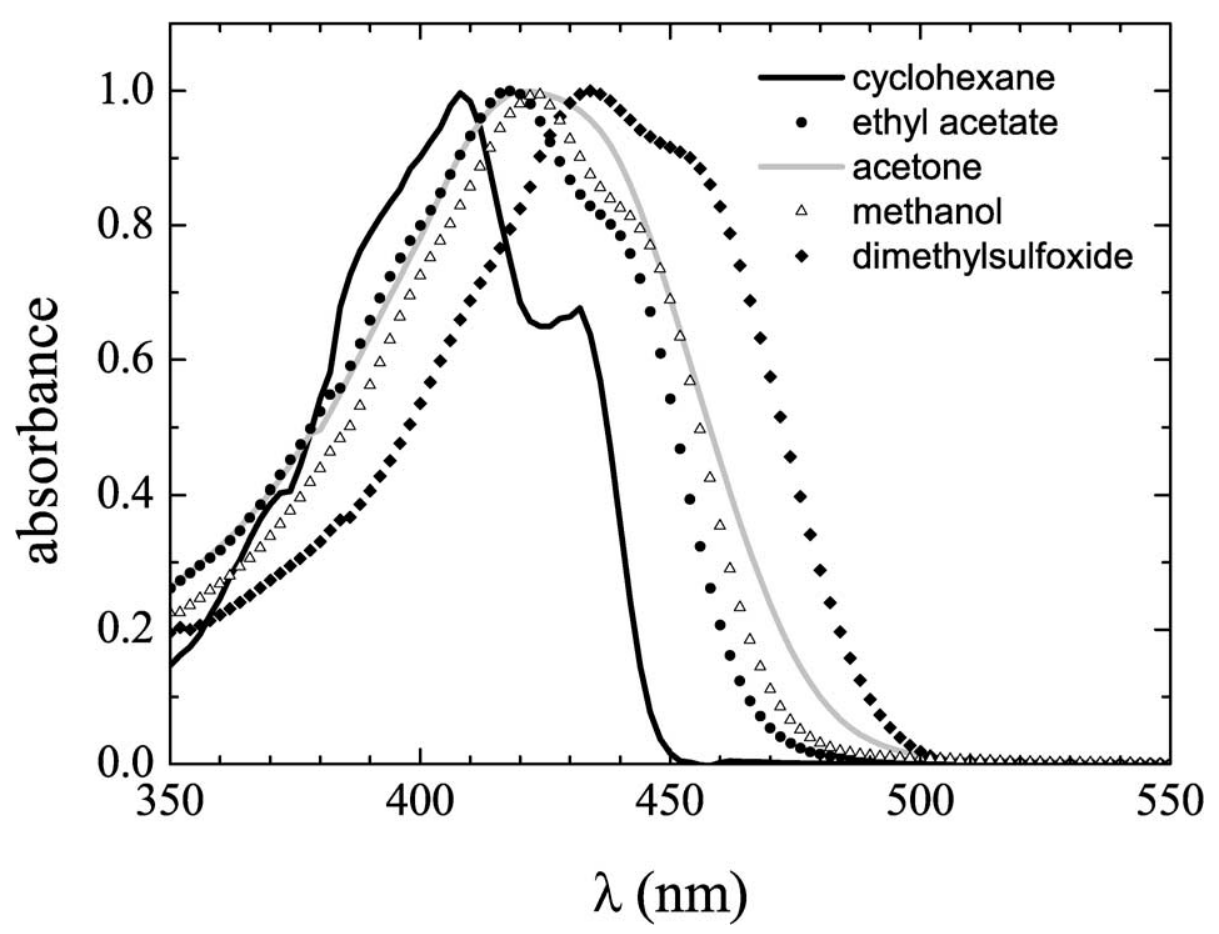

Fig. 2. Absorption spectra of curcumin in: cyclohexane (solid black line), ethyl acetate (dots), acetone (solid grey line), methanol (triangles), dimethyl-sulfoxide (diamonds). The peak absorbances were normalized to 1 .

\subsection{Fluorescence emission spectra}

Both the fluorescence intensity and the position of the fluorescence maxima, $\lambda_{\mathrm{Fl}}$, are much more sensitive to the nature of the solvent as compared to absorption (see the $\lambda_{\mathrm{Fl}}$ values in Table 1 and Fig. 3). The central fluorescence peak is detected at approximately $470 \mathrm{~nm}$ in cyclohexane, while it is progressively red shifted in polar non-protic solvents, where the $\lambda_{\mathrm{Fl}}$ values range between $494 \mathrm{~nm}$ in ethyl acetate and $521 \mathrm{~nm}$ in acetonitrile, and in H-bonding solvents. In particular, the alcohols (H-bond donors) induce larger red shifts ( $\lambda_{\mathrm{Fl}}$ being up to $566 \mathrm{~nm}$ in methanol and ethylene glycol) than H-bond acceptors $\left(\lambda_{\mathrm{Fl}}=536 \mathrm{~nm}\right.$ in DMFA and $\lambda_{\mathrm{Fl}}=550 \mathrm{~nm}$ in DMSO). The fluorescence spectral profiles are broad and essentially structureless. Cyclohexane is the only exception, as it displays a three-band structure (Fig. 3). The fluorescence quantum yield $\left(\Phi_{\mathrm{Fl}}\right)$ is generally low (see Table 1$)$. The highest values are found in polar non-protic solvents (ranging between $\Phi_{\mathrm{Fl}}=0.094$ in chloroform and $\Phi_{\mathrm{Fl}}=0.174$ in acetone) and the lowest one in cyclohexane $\left(\Phi_{\mathrm{Fl}}=0.006\right)$. Both $\mathrm{H}$-bond accepting and H-bond donating solvents produce intermediate $\Phi_{\mathrm{Fl}}$ values, which are very similar to each other (ranging between $\Phi_{\mathrm{Fl}}=0.022$ in ethylene glycol and $\Phi_{\mathrm{Fl}}=0.041$ in DMFA), except for the much higher value measured in isopropanol $\left(\Phi_{\mathrm{Fl}}=0.114\right)$.

\subsection{Excited-state dynamics}

The fluorescence decay times $\tau_{i}$ together with the relative initial amplitudes (see $A_{i}$, in brackets) are reported in Table 1.

Three decay components are identified in cyclohexane. Two fast components, with decay times $\tau_{1}=57 \mathrm{ps}$ and $\tau_{2}=256 \mathrm{ps}$, account for $99 \%$ of the fluorophores ( $83 \%$ and $16 \%$, respectively), while 


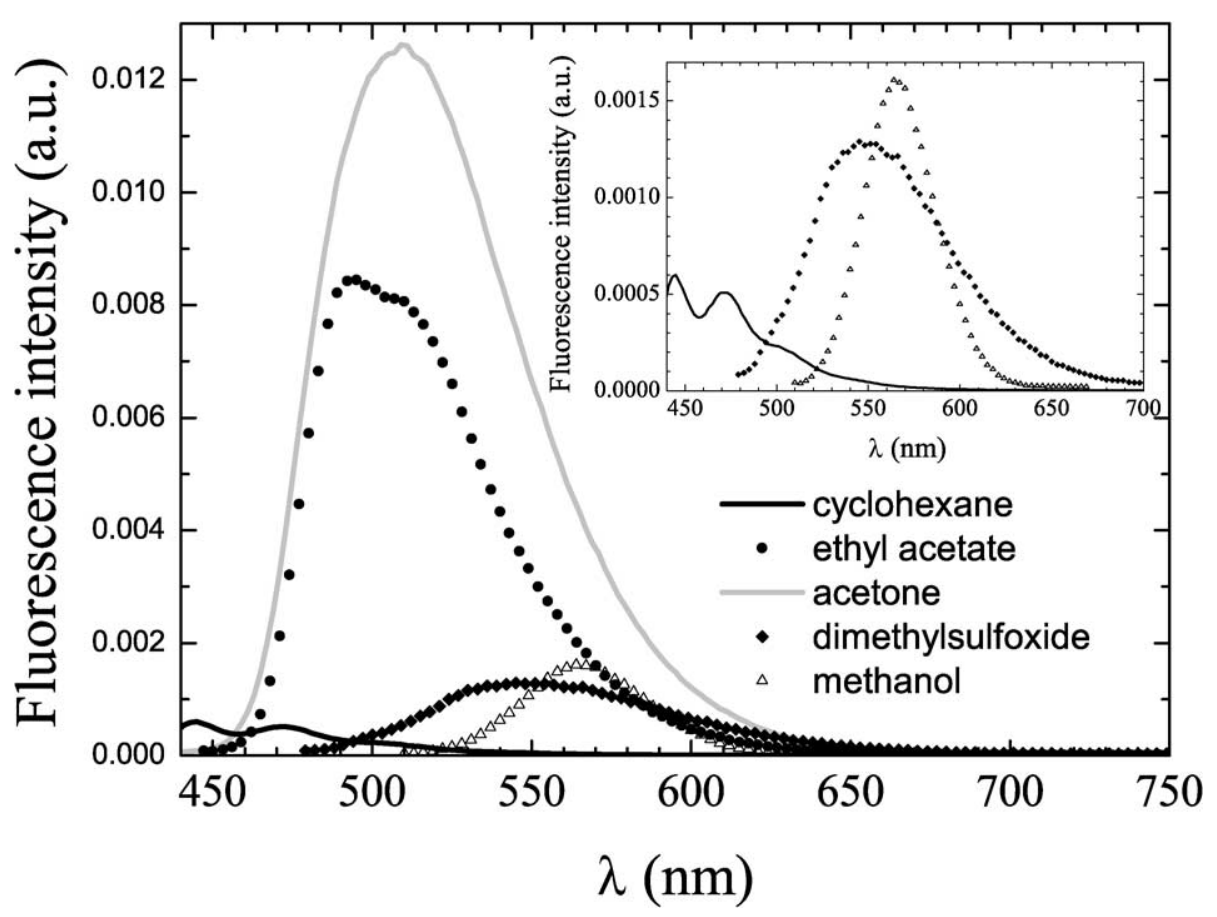

Fig. 3. Fluorescence spectra of curcumin in: cyclohexane (solid black line), ethyl acetate (dots), acetone (solid grey line), dimethyl-sulfoxide (diamonds), methanol (triangles). The spectra were corrected for the samples absorbance and normalized to a unit value of the ethyl acetate solution integrated fluorescence intensity.

a much slower component with decay time $\tau_{1}=1405$ ps accounts for the remaining $1 \%$. At difference, single exponential decays are observed in polar, non-protic solvents. The decays are essentially biexponential in the polar, H-bond accepting solvents. In alcohols, except isopropanol, in which a single exponential decay is observed, the decays are biexponential. The average fluorescence lifetime $\tau_{\mathrm{av}}$ (i.e. $\tau_{\mathrm{av}}=\sum_{i} \tau_{i} A_{i} / \sum_{i} A_{i}$ ) was calculated for each solvent by disregarding the decay components with $A_{i}<5 \%$. The $\tau_{\mathrm{av}}$ values are reported in Table 1. The trends of $\tau_{\mathrm{av}}$ and of $\Phi_{\mathrm{Fl}}$ are similar: the longest $\tau_{\mathrm{av}}$ values are those obtained for polar non-protic solvents (ranging between $\tau_{\mathrm{av}}=467 \mathrm{ps}$ in ethyl acetate and $\tau_{\mathrm{av}}=702 \mathrm{ps}$ in acetone) and the shortest one that for cyclohexane ( $\left.\tau_{\mathrm{av}}=89 \mathrm{ps}\right)$. In both H-bond accepting and H-bond donating solvents $\tau_{\mathrm{av}}$ (between $\tau_{\mathrm{av}}=155 \mathrm{ps}$ in DMSO and $\tau_{\mathrm{av}}=260 \mathrm{ps}$ in ethanol) is shorter than in polar non-protic solvents, but longer than in cyclohexane. Once again isopropanol is an exception ( $\tau_{\mathrm{av}}=476 \mathrm{ps}$, a value similar to those found for polar non-protic solvents).

By using the $\Phi_{\mathrm{Fl}}$ values and the corresponding $\tau_{\mathrm{av}}$ values displayed in Table 1, the rate constants for the radiative $\left(k_{\mathrm{Fl}}\right)$ and non-radiative $\left(k_{\mathrm{NR}}\right)$ decay can be calculated by means of the equations:

$$
\begin{aligned}
& k_{\mathrm{Fl}}=\frac{\Phi_{\mathrm{Fl}}}{\tau_{\mathrm{av}}}, \\
& k_{\mathrm{NR}}=\frac{1}{\tau_{\mathrm{av}}}-k_{\mathrm{Fl}} .
\end{aligned}
$$

The obtained values of $k_{\mathrm{Fl}}$ and $k_{\mathrm{NR}}$ are reported in Table 1 . The values of $k_{\mathrm{NR}}$ are substantially higher than the corresponding $k_{\mathrm{Fl}}$ values in all solvents. The $k_{\mathrm{NR}}$ values were of the order of $1-16 \mathrm{~ns}^{-1}$, while 
the $k_{\mathrm{Fl}}$ values were lower by factors between 5 (see acetonitrile) and 170 (see cyclohexane). Thus, the deactivation of $S_{1}$ occurs predominately via non-radiative pathways. The lowest $k_{\mathrm{NR}}$ values are those for polar non-protic solvents while the highest one is that for cyclohexane.

The quantum yield of photodegradation, $\Phi_{\text {Degr }}$, was measured in acetonitrile and methanol and its values are in Table 1 . We observe that $\Phi_{\mathrm{Degr}} \cong 0.5 \Phi_{\mathrm{Fl}}$ in acetonitrile whereas $\Phi_{\mathrm{Degr}} \cong \Phi_{\mathrm{Fl}}$ in methanol.

When we measured the efficiency of curcumin as a singlet oxygen photosensitizer we attained barely detectable phosphorescence signals in both acetonitrile and methanol.

\section{Discussion}

In order to explain the photophysics of a symmetric $\beta$-diketone such as curcumin, one should take into account the three diketo conformers and six enol conformers [13]. However, in solution curcumin mainly adopts the cis enol form (Fig. 1A) $[9,14,15]$ as confirmed by the lack of structures in both the absorption and fluorescence spectra. The untwisting of the aliphatic chain of the cis enol conformer in the ground state is ascribed to formation of a particularly strong intramolecular H-bond (Fig. 1C) between the hydroxyl and keto groups [16]. The anti diketo isomer (Fig. 1B), whose dipole moment has been calculated to be much smaller than that of cis enol, should not be ruled out in the case of non-polar solvents [14] even if the enol form dominates in both polar and non-polar solvents at room temperature [17]. In this frame, the two-band structure that we detected in the absorption spectrum in cyclohexane, in agreement with a similar observation in toluene made by Chignell et al. [9] could be indicative of the presence of both the cis enol and the anti diketo conformers. Increasing the solvent polarity results in shifting the keto $\leftrightarrow$ enol equilibrium towards the enol conformer, and to the loss of spectral structure. Structureless spectra similar to ours were measured in ethanol and acetonitrile by Chignell and coworkers [9].

Intermolecular $\mathrm{H}$-bonding with solvent molecules perturbs the intramolecular hydrogen bond characterizing the cis enol conformer. In the enol tautomer, H-bond donating solvents interact with the keto moiety, while H-bond acceptors interact with the enol proton (see Fig. 4(a)). Perturbation by polar,<smiles>[R]O[Hg]OC([R])=CC([R])=O</smiles><smiles>[R]O[Ga]O[Na]</smiles>

(a)

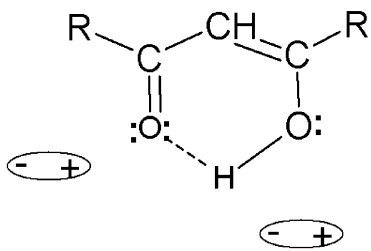

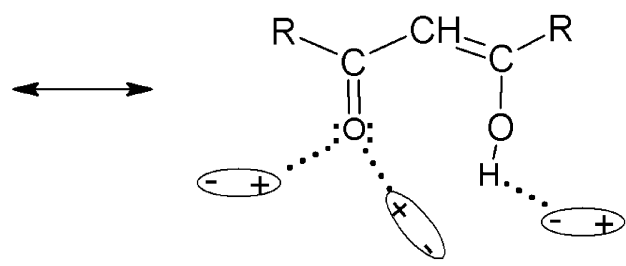

(b)

Fig. 4. (a) Perturbation of intramolecular H-bonding by protic solvents; (b) perturbation of intramolecular H-bonding by polar, non-protic solvents. 
non-protic solvents is also likely to occur by polarity effects (Fig. 4(b)). As the weakening of the intramolecular H-bond makes the curcumin structure less rigid and more prone to out-of-plane vibration, we expect, and actually observe (see $\lambda_{\mathrm{Abs}}$ and $\lambda_{\mathrm{Fl}}$ data in Table 1), the smallest Stokes shift in cyclohexane. For the same reason, the much higher Stokes shifts that we find in both non-protic and protic polar solvents can be taken as indication that both polarity effects and intermolecular H-bond formation heavily perturb intramolecular $\mathrm{H}$-bonding.

In H-bonding solvents, besides the cis conformer, substantial amounts of trans enol have been reported for simple $\beta$-diketones (Fig. 1A). The enhanced stabilization induced by the formation of intermolecular $\mathrm{H}$-bonds with solvent molecules, which is impossible in the cis conformer for steric reasons, seems to be sufficient to shift the equilibrium to the trans enol, whose presence might correlate with the appearance of shoulders in the absorption spectra of curcumin in the protic solvents.

Instauration of the intramolecular H-bond in the cis enol conformer brings about new mechanisms that might influence the internal conversion rate and affect $k_{\mathrm{NR}}$. Hydrogen-stretching vibrations, e.g. a lengthening of the $\mathrm{O}-\mathrm{H}$ bond and a shortening of the hydrogen bond in the cis enol form of $S_{1}$, could lead to radiationless decay. As we observe minimum Stokes shift in the solvent for which the H-bond is the tightest (cyclohexane, see also toluene [9]) deactivation by $\mathrm{H}$-stretching vibrations is apparently not efficient. Charge-transfer interactions provide other radiationless decay pathways to the cis enol conformer. Three proton transfer mechanisms can occur: exchange between the vinylic $\mathrm{CH}$ and the enol $\mathrm{OH}$ sites (i.e. enol $\leftrightarrow$ diketo tautomerism), intermolecular exchange of the labile $\mathrm{OH}$ proton, and its transfer from one oxygen to the other of the cis enol conformer. The last mechanism is by far the fastest one and hence the most relevant [13]. This excited-state intramolecular proton transfer (ESIPT) becomes even faster in the presence of the intramolecular H-bond in Fig. 1C [18-21], as demonstrated for molecules having chemical structures similar to that of curcumin. Rates as high as $10^{11} \mathrm{~s}^{-1}$ were reported [19]. As curcumin has both electron-donating and electron-accepting properties, in protic environments $S_{1}$ deactivation by intermolecular electron transfer should also be considered [14]. On the contrary, intramolecular electron transfer can be ruled out as the molecule lacks both donor and acceptor groups [22].

Electronically excited $\beta$-diketones can also deactivate by undergoing photochemical re-ketonization $[23,24]$. The process accompanying the enol excitation is postulated to start with cis-trans isomerization, so that the intramolecular H-bonding stabilization of the cis enol is lost, followed by decay via either proton transfer to the vinylic $\mathrm{CH}$ (re-ketonization) or recovery of the H-bonded cis enol conformer and subsequent ESIPT (Fig. 5) [23]. The first $S_{1}$ deactivation mechanism generates an anti diketo conformer in its ground state, and it is thus favored in non-polar solvents, while the second one generates a cis enol conformer stabilized by its intramolecular H-bond, and is most likely to occur in polar solvents.

As to the decay pathways intersystem crossing and photodecomposition, our results on singlet oxygen generation and photodegradation indicate that, at least in polar solvents (be they protic or not), none of them is relevant.

All $\Phi_{\mathrm{Fl}}$ and $\tau_{\mathrm{av}}$ data in Table 1, together with the calculated $k_{\mathrm{Fl}}$ and $k_{\mathrm{NR}}$, show that the non-radiative pathways are most efficient in cyclohexane, where the tightness of intramolecular H-bond ensures the occurrence of ESIPT. This is a signal that ESIPT is the leading mechanism in curcumin decay. However, both the three-exponential fluorescence decay and the three-band fluorescence spectrum indicate the existence of other mechanisms with comparable rates. We associate the shortest decay, and the least Stokes shifted fluorescence peak, to the cis enol curcumin molecules excited to the fluorescent state without breaking the H-bond. The intermediate decay, and the fluorescence band peaked at $471 \mathrm{~nm}$, can be associated to the cis enol conformers that experience enol cis-trans isomerization upon excitation and 
<smiles>[R]C1=CC([R])OCO1</smiles>

$\mathrm{H}$ bonded cis-enol<smiles>[R]C(=O)CC([R])=O</smiles>

Fig. 5. Photochemically induced re-ketonization.

deactivate by re-ketonization, while the very few molecules emitting the longest-lived fluorescence are the ground-state anti diketo conformers, that cannot undergo ESIPT. It is also reasonable to associate these conformers to the fluorescence band peaking at $502 \mathrm{~nm}$, which is the most Stokes shifted due to their flexibility with respect to out-of-plane vibrations.

The $k_{\mathrm{NR}}$ values in Table 1 are smaller in protic solvents than in the non-polar solvent. As stated above, the fluorescence decays in all the protic solvents, except isopropanol, are biexponential. The fastest decay time values are not of the order of few tens of picoseconds, as expected for direct ESIPT driven decays, but approximately 200 ps. Possibly, the instauration of intermolecular H-bonds results in the almost complete intramolecular H-bond breaking, with consequent blocking of direct ESIPT. Proton transfer can no longer occur directly due to the lack of any electronic connection between the separate solvated groups. Desolvation is required to form the fast-decaying intramolecular $\mathrm{H}$-bonded enol structure. Because the ESIPT step is rapid once an intramolecular H-bond is formed, the decay time observed for this deactivation path will depend essentially on the rate of the desolvation process. Anyway, alternative decay mechanisms involving delivery of the excitation energy to the solvent molecules are expected to be highly enhanced by the instauration of intermolecular H-bonds. On the contrary, even if a large number of excited trans enol conformers should be found in protic solvents, re-ketonization is not probable, as the anti diketo conformer is much less polar as compared to the two enol tautomers. Biexponential fluorescence decays can be thus explained by hypothesizing a fast, intermolecular H-bond mediated, $S_{1}$ deactivation by transfer of energy to the solvent molecules and a slow, solvent-reorganization moderated, ESIPT. In the case of isopropanol, the first mechanism might be somehow prevented.

The $\Phi_{\mathrm{F} 1}$ values are the highest and the $\tau_{\mathrm{av}}$ values the longest in polar non-protic solvents. The $k_{\mathrm{Fl}}$ are comparable to those obtained in the case of protic solvents, but the $k_{\mathrm{NR}}$ are three to five times 
slower. This indicates that also polar non-protic solvents efficiently inhibit deactivation through ESIPT, but, at difference with protic solvents, their interactions with curcumin do not bring about new decay mechanisms. This hypothesis is supported by the single exponential decays we observed in all polar nonprotic solvents. Interestingly, the $\tau_{1}$ values measured in the non-protic solvents are in the same range as those of the longer decay times, $\tau_{2}$, in protic solvents, that we associated to molecules decaying through solvent-rearrangement moderated ESIPT.

\section{Conclusion}

The excited state behavior of curcumin is strongly dependent on the reaction medium. We could successfully explain the features of the spectroscopic data we measured by assuming three simple hypothesis, that were previously postulated by other authors:

(1) Curcumin at room temperature is virtually found in only its cis enol conformer $[9,14,15,17]$. Lower amounts of the anti diketo and the trans enol conformers are also found in non-polar [14] and protic environment, respectively.

(2) ESIPT is the leading non-radiative decay mechanism of $S_{1}[13,19]$.

(3) The ESIPT rate is higher the tighter is the intramolecular H-bond between the enol and the keto moiety [18-21].

The above results may be of help in optimizing drug delivery systems suitable for the administration of curcumin to patients in view of its future clinical application as a photosensitizer.

\section{References}

[1] H.H. Tønnesen, Studies on curcumin and curcuminoids. XVIII. Evaluation of curcuma products by use of standardized reference color values, Z. Lebensm. Unters. Forsch. 194 (1992), 129-130.

[2] B.B. Aggrawal, C. Sundaram, N. Malani and H. Ichikawa, Curcumin: the Indian solid gold, Adv. Exp. Med. Biol. 595 (2007), 1-75.

[3] A. Duvoix, R. Blasius, S. Delhalle, M. Schnekenburger, F. Morceau, E. Henry, M. Dicato and M. Diederich, Chemopreventive and therapeutic effects of curcumin, Cancer Lett. 223 (2005), 181-190.

[4] M.E. Egan, M. Pearson, S. Weiner, V. Rajendran, D. Rubin, J. Glockner-Pagel, S. Canny, K. Du, G.L. Lukacs and M.J. Caplan, Curcumin, a major constituent of turmeric, corrects cystic fibrosis defects, Science 304 (2004), 600-602.

[5] Z. Sui, R. Salto, J. Li, C. Craik and P.R. Ortiz, Inhibition of the HIV-1 and HIV-2 proteases by curcumin boron complexes, Bioorg. Med. Chem. 1 (1993), 415-422.

[6] E. Bruzell, E. Morisbak and H.H. Tønnesen, Studies on curcumin and curcuminoids. XXIX. Photoinduced cytotoxicity of curcumin in selected aqueous preparations, Photochem. Photobiol. Sci. 4 (2005), 523-530.

[7] K.C. Das and C.K. Das, Curcumin (diferuloylmethane), a singlet oxygen $\left({ }^{1} \mathrm{O}_{2}\right)$ quencher, Biochem. Biophys. Res. Com. 295 (2002), 62-66.

[8] A.A. Gorman, I. Hamblett, V.S. Srinivasan and P.D. Wood, Curcumin-derived transients: a pulsed laser and pulse radiolysis study, Photochem. Photobiol. 59 (1994), 389-398.

[9] C.F. Chignell, P. Bilski, K.J. Reszka, A.G. Motton, R.H. Sik and T.A. Dahl, Spectral and photochemical properties of curcumin, Photochem. Photobiol. 59 (1994), 295-302.

[10] A. Tomren, M. Màsson, T. Loftsson and H.H. Tønnesen, Studies on curcumin and curcuminoids. XXXI. Symmetric and asymmetric curcuminoids: stability, activity and complexation with cyclodextrin, Int. J. Pharm. 338 (2007), 27-34.

[11] R. Velapoldi and H.H. Tønnesen, Corrected emission spectra and quantum yields for a series of fluorescent compounds in the visible spectral region, J. Fluoresc. 14 (2004), 465-472.

[12] D.E. Moore, Standardization of kinetic studies and photodegradation reactions, in: Photostability of Drugs and Drug Formulations, 2nd edn, H.H. Tønnesen, ed., CRC Press, Boca Raton, 2004, pp. 49-53. 
[13] J. Emsley, The composition, structure and hydrogen bonding of the $\beta$-diketones, in: Structure and Bonding, M.J. Clarke, J.B. Goodenough, J.A. Ibers, C.K. Jørgensen, D.M.P. Mingos, J.B. Neilands, D. Reinen, P.J. Sadler, R. Weiss and R.J.P. Williams, eds, Springer-Verlag, Berlin, 1984, pp. 148-191.

[14] K. Balasubramanian, Molecular orbital basis for yellow curry spice curcumin's prevention of Alzheimer's disease, J. Agric. Food Chem. 54 (2006), 3512-3520.

[15] U. Pedersen, P.B. Rasmussen and S.O. Lawesson, Synthesis of naturally occurring curcuminoids and related compounds, Liebigs Ann. Chem. 1985 (1985), 1557-1569.

[16] J.T. Mague, W.L. Alworth and F.L. Payton, Curcumin and derivatives, Acta Cryst. C 60 (2004), 608-610.

[17] F. Ortica and M.A.J. Rodgers, A laser flash photolysis study of curcumin in dioxane-water mixtures, Photochem. Photobiol. 76 (2001), 745-751.

[18] S.R. Flom and P.F. Barbara, Proton transfer and hydrogen bonding in the internal conversion of $\mathrm{S}_{1}$ anthraquinones, J. Phys. Chem. 89 (1985), 4489-4494.

[19] A.J.G. Strandjord, S.H. Courtney, D.M. Friedrich and P.F. Barbara, Excited-state dynamics of 3-hydroxyflavone, J. Phys. Chem. 87 (1983), 1125-1133.

[20] S. Mitra and S. Mukherjee, Photophysical properties of substituted intramolecularly hydrogen bonded compounds: A combined experimental and theoretical study, J. Lumin. 118 (2006), 1-11.

[21] P.H. Bong, Spectral and photophysical behaviours of curcumin and curcuminoids, Bull. Korean Chem. Soc. 21 (2000), 81-86.

[22] Z.R. Grabowski and K. Rotkiewicz, Structural changes accompanying intramolecular electron transfer: focus on twisted intramolecular charge-transfer states and structures, Chem. Rev. 103 (2003), 3899-4031.

[23] A.C. Weedon, Photochemical reactions involving enols, in: The Chemistry of Enols, Z. Rappoport, ed., John Wiley \& Sons, New York, 1990, pp. 591-638.

[24] P. Nikolov, F. Fratev, I. Petkov and P. Markov, Dimer fluorescence of some $\beta$-dicarbonyl compounds, Chem. Phys. Lett. 83 (1981), 170-173. 


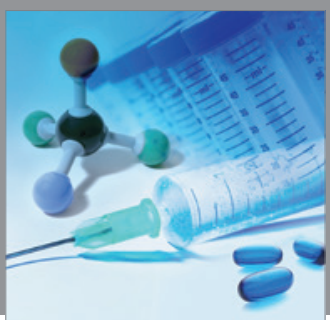

International Journal of

Medicinal Chemistry

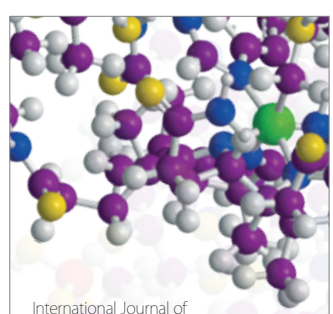

Carbohydrate Chemistry

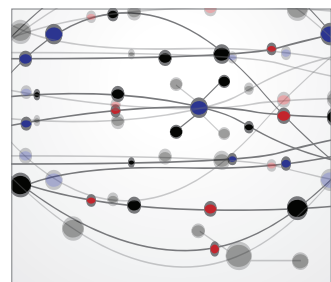

The Scientific World Journal
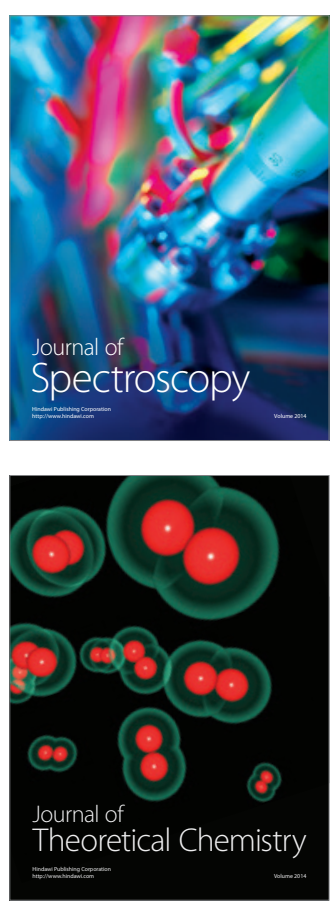
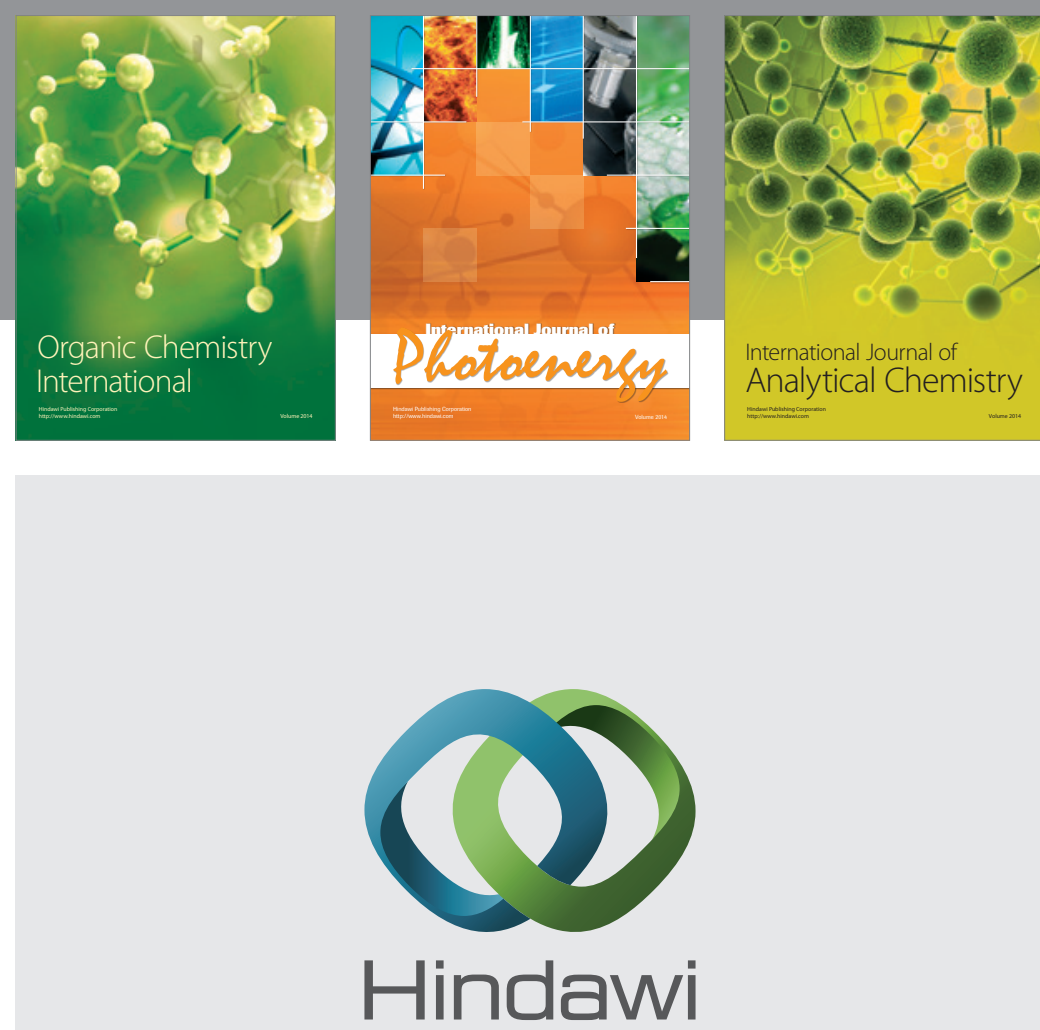

Submit your manuscripts at

http://www.hindawi.com
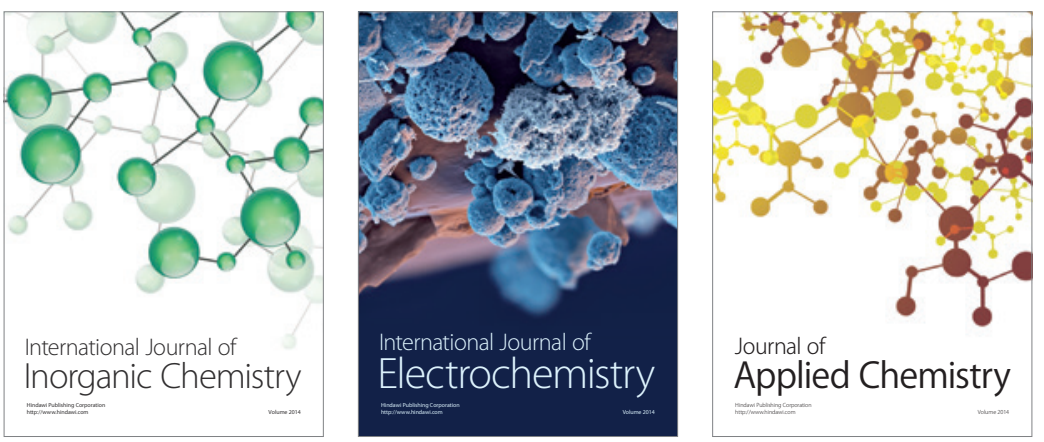

Journal of

Applied Chemistry
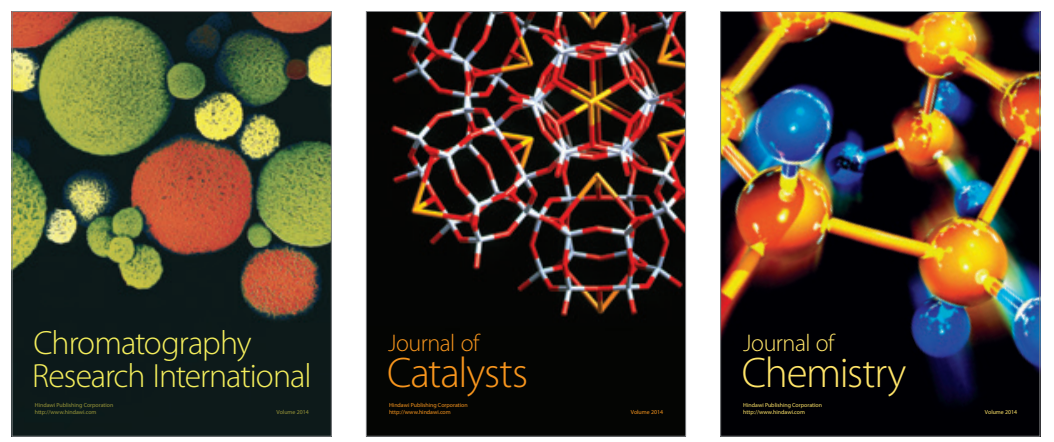
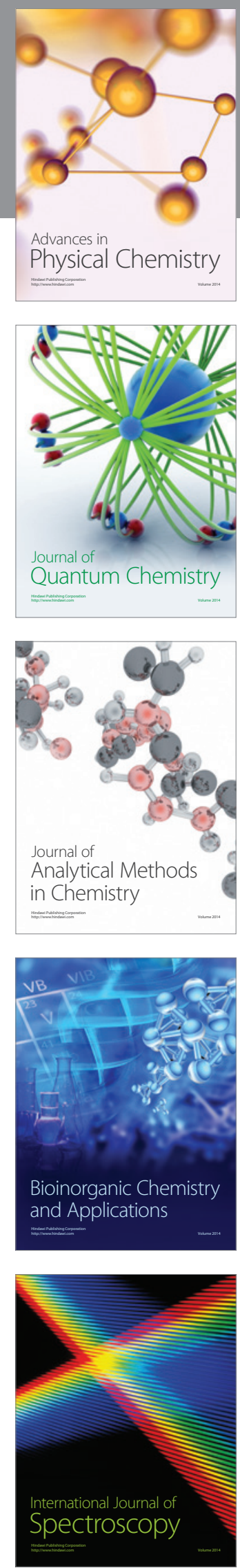